\title{
Band Gaps and Single Scattering of Phononic Crystal*
}

\author{
Xiaoyi Huang ${ }^{1 \#}$, Jingcui Peng ${ }^{2}$, Huanyou Wang ${ }^{1}$, Gui Jin ${ }^{1}$ \\ ${ }^{1}$ Department of Physics, Xiangnan University, Chenzhou, China \\ ${ }^{2}$ Department of Applied Physics, Hunan University, Changsha, China \\ E-mail: ${ }^{j}$ ghxy@126.com \\ Received August 28, 2011; revised September 29, 2011; accepted October 11, 2011
}

\begin{abstract}
A method is introduced to study the transmission and scattering properties of acoustic waves in two-dimensional phononic band gap (PBG) materials. First, it is used to calculate the transmission coefficients of PBG samples. Second, the transmitted power is calculated based on the far field approach. We have also calculated the scattering cross section, the results indicate that phononic band gap appear in frequency regions between two well separated resonance states.
\end{abstract}

Keywords: Phononic Crystal, Band Gap, Single Scattering

\section{Introduction}

The acoustic properties of a locally homogeneous and isotropic composite material is characterized by a set of parameters varying in space: mass density $\rho$, Lamé coefficients $\lambda$, and $\mu$. In this paper we focus on the composite materials, which consist of homogeneous particles distributed periodically in a host medium. They are characterized by different mass densities and Lamé coefficients. When identical particles are distributed periodically in a host medium, the composite material may be referred to as a phononic crystal. Recently the propagation of elastic or acoustic waves (EL or AC wave) in a phononic crystal has received much renewed attention [1-9]. These new materials can be of real interest since a large contrast between the elastic parameters is allowed. For example, systems composed of very soft rubber [10] are more likely to obtain the low-frequency gaps with a structure of small dimension. This can lead to promising applications such as a low-frequency vibration/noise devices such as lenses and acoustic interferometers [11]. On the other hand, more sophisticated combinations such as fluids infiltrated in a drilled solid [5] or solid-solid systems [7] have been demonstrated to produce a full phononic band gap for ultrasounds. Phononic crystal make possibility of the achievement of complete frequency band gaps that are useful to prohibit specific vibrations in accurate technologies such as transducers and sonar.

In the plane-wave expansion method, the finite differ-

*Supported by the Key Project of Education Department of Hunan Province (09A086), Science and Technology Project of Chenzhou. ence time domain and the multiple-scattering theory are commonly used in order to study the elastic response of phononic crystal [12-15]. In this work, in order to study the propagation of acoustic waves in phononic crystal, we consider a two-dimensional periodic system consisting of finite cylinders of circular cross section. The system is periodic in the $x-y$ plane and within it there is a translational invariance in the direction (z) parallel to the cylinders. The intersection of the cylinders with a transverse plane makes a square lattice. We treated finite PBG samples as scattering objects in open geometry, The radiation boundary condition was naturally imposed. Considering the far-field approach, we have independently adopted this method to study the transmission and scattering properties of finite PBG samples. In the case of transmission, a generalized transmission coefficient can be defined in terms of the far-field total scattering amplitude, from the total scattering amplitude we can retrieve the dispersion relations and the decay length inside a gap. By adopted this method, the incident field, scattered field and the total scattering amplitude become very simple form, the calculating can be extremely simplified. We explicitly demonstrate that this method can produce transmission results that are in excellent quantitative agreements with the available experimental data.

\section{Model and Formula}

The displacement vector $\boldsymbol{U}(\boldsymbol{r}, t)$ in a homogeneous elastic medium of mass density $\rho$ and Lamé coefficients $\lambda, \mu$ satisfies the following equation: 


$$
(\lambda+2 \mu) \nabla(\nabla \cdot \boldsymbol{U})-\mu \nabla \times(\nabla \times \boldsymbol{U})=\rho \partial_{t}^{2} \boldsymbol{U}=0
$$

In the case of a harmonic elastic wave with angular frequency $\omega$, we have

$$
\boldsymbol{U}(\boldsymbol{r}, t)=\operatorname{Re}[\boldsymbol{U}(\boldsymbol{r}) \exp (i \omega t)]
$$

and Equation (1) was reduced to the following time-independent form

$$
\omega(\lambda+2 \mu) \nabla(\nabla \cdot \boldsymbol{u})-\mu \nabla \times(\nabla \times \boldsymbol{u})+\rho \omega^{2} \boldsymbol{u}=0 .
$$

Defining

where

$$
\boldsymbol{u}=\boldsymbol{I}+\boldsymbol{m}+\boldsymbol{n},
$$

where $\boldsymbol{Z}$ is the unit vector along the $\mathrm{z}$-axis. $\varphi, \chi$ and $\psi$ are the displacement potential functions of longitudinal and two transverse waves respectively. The displacement potential function of the incident longitudinal waves can be expanded in terms of the cylindrical Bessel Function [16]

$$
\varphi_{\text {inc }}=\exp \left(j k_{z} z\right) \sum_{-\infty}^{\infty}(j)^{n} J_{n}\left(k_{l r} r\right) \exp (j n \theta),
$$

where $k_{l r}\left(=k_{l}^{2}-k_{z}^{2}\right)^{1 / 2}$ is the radial component of the incident wave vector, $J_{n}$ is the Bessel function of the first kind of order $n, k_{z}$ is the $\mathrm{z}$-axis component of the incident wave vector; $k_{l}$ is the longitudinal wave numbers, $r$ is the normal distance of the field spot away from z-axis; and $\theta$ is the angle of direction.

The displacement potential functions of the longitudinal and transverse scattered waves can also be expanded:

$$
\begin{aligned}
& \varphi_{s c}=\exp \left(j k_{z} z\right) \sum_{n=-\infty}^{\infty} A_{n} H_{n}\left(k_{l r} r\right) \exp (j n \theta), \\
& \psi_{s c}=\exp \left(j k_{z} z\right) \sum_{n=-\infty}^{\infty} B_{n} H_{n}\left(k_{l r} r\right) \exp (j n \theta), \\
& \chi_{s c}=\exp \left(j k_{z} z\right) \sum_{n=-\infty}^{\infty} C_{n} H_{n}\left(k_{l r} r\right) \exp (j n \theta),
\end{aligned}
$$

where $H_{n}$ is the Hankel function. Using the same method we can expand the displacement potential functions of the incident transverse waves in terms of the cylindrical Bessel functions. Therefore the displacement potential functions of the incident transverse waves inside the cylinders are expanded as:

$$
\begin{aligned}
\varphi_{i n} & =\exp \left(j k_{z} z\right) \sum_{n=-\infty}^{\infty}\left\{\left[A_{n} H_{n}\left(k_{l r} r\right)+D_{n} \delta_{n 0}\right]\right. \\
& \left.+\left[B_{n} J_{n}\left(k_{t r} \cdot r\right)+E_{n} \delta_{n 0}\right]\right\} \exp (j n \theta)
\end{aligned}
$$

$$
\begin{aligned}
& \psi_{\text {in }}=\exp \left(j k_{z} z\right) \sum_{n=-\infty}^{\infty}\left\{\left[A_{n} J_{n}\left(k_{l r} \cdot r\right)+D_{n} \delta_{n 0}\right]\right. \\
&\left.+\left[B_{n} J_{n}\left(k_{t r} \cdot r\right)+E_{n} \delta_{n 0}\right]\right\} \exp (j n \theta) \\
& \chi_{\text {in }}=\exp \left(j k_{z} z\right) \sum_{n=-\infty}^{\infty}\left[C_{n} J_{n}\left(k_{l r} \cdot r\right)+F_{n} \delta_{n 0}\right] \exp (j n \theta)(
\end{aligned}
$$

where $A_{n}-F_{n}$ are coefficients and $k_{t r}=\left(k_{t}^{2}-k_{z}^{2}\right)^{1 / 2}$.

In the following we consider a sample of the two-dimensional periodic arrays system. The sample was made of d-radius rods with lattice constant a. The position of the rod with index $j$ corresponds to $\boldsymbol{r}_{j}=\left(r_{j}, \theta_{j}\right)$. What are around this rod are incident waves involving external sources and scattered waves from other rods. The total field around this rod is $u=u_{\text {inc }}+u_{\text {scatt }}$.

The coefficients $A_{n}-F_{n}$ are defined depending on the boundary conditions.

In the light of the continuity of the displacements, there are

$$
\left.u_{i}^{i n c}\right|_{r=d}+\left.u_{i}^{s c}\right|_{r=d}=\left.u_{i}^{i n}\right|_{r=d} \quad i:(r, \theta, z),
$$

Due to the continuity of the stresses, there exists:

$$
\left.p_{i}^{i n c}\right|_{r=d}+\left.p_{i}^{s c}\right|_{r=d}=\left.p_{i}^{i n}\right|_{r=d} \quad i:(r, \theta, z),
$$

where

$$
p_{i}=\sum_{j} \sigma_{i j} n_{j} \quad i, j:(r, \theta, z)
$$

and $\sigma_{i j}=2 \rho c_{t}^{2} u_{i j}+\rho\left(c_{t}^{2}-2 c_{t}^{2}\right) \delta_{i j} \sum_{l} u_{l l} \quad i, j, l:(r, \theta, z)$.

where $\sigma_{i j}$ are the stress tensor elements and $u_{i j}$ are the strain tensor elements that result from the components of the displacement vector. The superscripts inc, sc, in, denote the incident, the scattered and the inner field respectively.

In the far field, when $k_{l} r\left(k_{t} r\right) \gg 1$, $u_{\text {scatt }}(\boldsymbol{r}) \rightarrow f_{s}(\theta) \exp (i k r) / \sqrt{r}$.

The total scattering amplitude of the longitudinal waves from Equations (9)-(11) is

$$
f_{s}(\theta)=\frac{2}{\sqrt{\pi k_{l}}}\left|\sum_{n=-\infty}^{\infty} j^{-n} A_{n}^{N} \exp (j n \theta)\right| .
$$

For acoustic wave transmission, a slit with width $\mathrm{w}$ along the $y$ direction is put between a source and the sample. Acoustic waves propagate along $x$ direction. In this case, the incident field can be obtained from the Kirchoff integral formula [17]:

$$
u_{\text {inc }}(x, y)=\left(\frac{k_{l}}{4}\right) \int_{-w / 2}^{w / 2} \mathrm{~d} y^{\prime}\left[H_{0}\left(k_{l} r^{\prime}+i \frac{x}{r^{\prime}} H_{1}\left(k_{l} r^{\prime}\right)\right)\right] \text {, }
$$

where $r^{\prime}=\sqrt{x^{2}+\left(y-y^{\prime}\right)^{2}}$, in the case of far field, 
when $r \gg w$, Equation (18) becomes

$$
\begin{aligned}
u_{i n c}(\boldsymbol{r}) & \rightarrow a_{0}(\theta) \exp \left(i k_{l} r\right) / \sqrt{r} \text { with } \\
a_{0}(\theta) & =\mathrm{e}^{-i \pi / 4} \sqrt{\frac{k_{l}}{2 \pi}} \cos ^{2}(\theta / 2) \frac{2 \sin \left[k_{l}(w / 2) \sin \theta\right]}{k_{l} \sin \theta} .
\end{aligned}
$$

The vector of energy flux density is:

$$
\boldsymbol{S}(\boldsymbol{r})=\frac{-c}{8 \pi k_{l}} \operatorname{Im}[u(\boldsymbol{r}) \nabla u *(\boldsymbol{r})] .
$$

Therefore the far-field energy flux has the form

$$
\boldsymbol{S}(\boldsymbol{r}, \theta)=\frac{c\left[\left|a_{0}(\theta)+f_{s}(\theta)\right|\right]}{8 \pi r} \widehat{r}=S(\theta) \frac{\widehat{r}}{r} .
$$

So we define a transmission coefficient as the ratio of transmission energy flux to that of the incident wave at $\theta=0$.

Therefore:

$$
T=\left|1+\frac{f_{s}(0)}{a_{0}(0)}\right|^{2}=\left|1+\sqrt{\frac{2 \pi}{k_{l} w^{2}}} \mathrm{e}^{i \pi / 4} f_{s}(0)\right|^{2} .
$$

According to Equation (17) and the definition of scattering cross section, the dimensionless scattering cross sections of the longitudinal and transverse scattered waves have the form:

$$
\begin{aligned}
& \hat{\sigma}_{l}=\frac{1}{k_{l} \cdot d} \sum_{n=-\infty}^{\infty} \delta_{n 0}\left(\left|a_{n}\right|^{2}+\left(\frac{c_{t 1}}{c_{l 1}}\right)^{2}\left|b_{n}\right|^{2}\right) \\
& \hat{\sigma}_{t}=\frac{1}{k_{t} \cdot d} \sum_{n=-\infty}^{\infty} \delta_{n 0}\left(\left(\frac{c_{l 1}}{c_{t 1}}\right)^{2}\left|a_{n}\right|^{2}+\left|b_{n}\right|^{2}\right)
\end{aligned}
$$

where $\hat{\sigma}_{l}$ and $\hat{\sigma}_{t}$ are the scattering cross sections for longitudinal and transverse incident wave respectively, $k_{l}$ and $k_{t}$ are the longitudinal and transverse wave numbers for the host, $c_{l 1}$ and $c_{t 1}$ are the longitudinal and transverse wave velocities for the host, $d$ is the diameter of cylinder, $a_{n}$ and $b_{n}$ are the longitudinal and transverse scattered wave coefficients.

For elastic media, there is a reasonable amount of calculations for infinite systems [18-20]. However, systems are finite and there are boundaries. Therefore under a proper choice of parameters, states sliding and propagating along the surface and localized in the normal to the surface, i.e., should appear, these are analogous to electronic surface in crystals [21] and to those calculated for photonic systems [22]. According to M. Torres et al. that surface state solutions are consubstantial with finite systems and exist for sonic propagation in finite elastic media. They deal with several realizations of structures for ultrasonic propagation in elastic media to observe such surface state modes and localization phenomena in linear and point defects [3]

\section{Numerical Results}

In this paper, the finite-sized PBG sample used in the calculation consisted of 6 rows along the $x$ axis, 36 column rods with steel rods arranged in air host as square with the lattice constant a, with filling fraction of $f=0.55$, rod radius mains $=0.35 a$, and a room of temperature $25^{\circ} \mathrm{C}$. The mass density of phononic crystal is $\rho=7800$ $\mathrm{g} / \mathrm{cm}^{3}$, longitudinal wave velocity $c_{l}=5940 \mathrm{~m} / \mathrm{s}$, transverse wave velocity $c_{t}=3220 \mathrm{~m} / \mathrm{s}$ [23], width of slit $w=$ $3.5 a$, and is placed at a distance of $l=2.1 a$. From Equation (22) we have calculated the transmission coefficient and total transmitted power as $P_{T}=2 \int_{0}^{\frac{\pi}{2}} s(\theta) d \theta$.

Figure 1 shows the calculated results of transmission coefficient and total transmitted power, in dimensionless frequency region (1.75 to 2.25). Acoustic wave propagation is inhibited forming frequency band gaps. The transmission coefficient curve is finite and total transmitted power becomes zero. The results are in excellent agreement with previous results from Ref. [1].

Taking $\mathrm{T} \approx 0$ in Equation (22) which gives $\left|f_{s}(0)\right| / \sqrt{a} \approx \sqrt{f}(w / a)$ inside the bands $\left|f_{s}(0)\right|$ show large variations in frequency. This is related to the phase shift of the scattered waves, if we assuming $T \approx 1$

$\left|f_{s}(0)\right| / a_{0}(0) \approx 2 i \sin (\Phi / 2) \exp [i \Phi / 2]$ and $\left|f_{s}(0)\right| / \sqrt{a} \approx 2|\sin (\Phi / 2)| \sqrt{f}(w / a)$, where $\Phi$ is the phase difference between outgoing and incoming waves. $\Phi$ changes rapidly near band edges. The derivative of $\Phi(f)$ gives information on group velocity $v_{g}$. At band edge, $\mathrm{d} \Phi / \mathrm{df}$ diverges and $v_{g}$ approaches zero. Therefore from $f_{s}(0)$ we are able to extract the effective elastic constant for frequencies inside a band and the decay length for frequencies inside a gap.

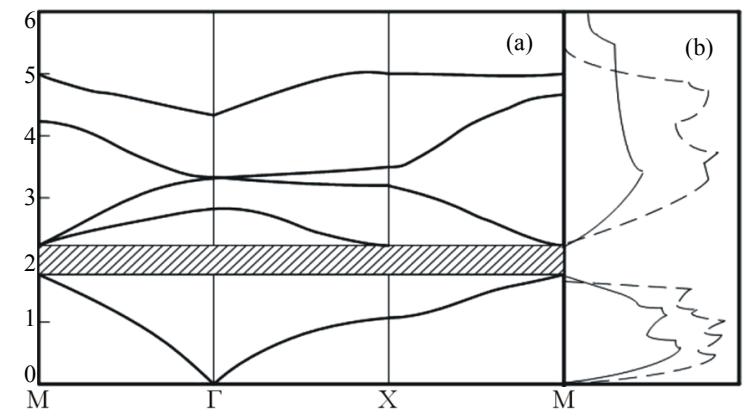

Figure 1. Acoustic band structure and transmission coefficients and total transmitted power for a square array of rigid stainless steel cylinders in air host. The filling fraction is $f=0.55$. (a) The band structures reproduced from Ref. [1]; (b) Solid curves: transmission coefficients. Dashed curves: total transmitted power. 
From Equation (23) and Equation (24) we have calculated the dimensionless scattering cross sections.

Figure 2 shows the calculated results of dimensionless scattering cross sections, The gap appears in $1.9 \leq k_{l} d\left(k_{t} d\right)$ $\leq 2$.8. The arrows denote the position of band gap, Figure 2(c) shows one full band gap. These results agree with Figure 1.

Here, we try to connect the appearance of a gap and other characteristics of the band structure in a periodic system consisting of cylinder inclusions in a homogeneous matrix with the form of the cross section from a single inclusion. This connection determines to what extent single scattering is an important factor in determining some characteristic features in the band structure, and how it can be used to predict the possible existence of gaps. For cylinder inclusions in a host material, the existence of full gaps has been connected to the following picture: There are two channels for propagation. One is mainly using the host material and the other is employing the resonance states. Coherent jumping from resonance state creates this second channel. In analogy, with the linear combination of atomic orbital (LCAO, otherwise called tight binding approximation) in the electronic band structure.

In attempting this extension of the LCAO approach to $\mathrm{AC}$, one should keep in mind some important differences between the two cases. Resonances are not true eigenstates, rigorously localized inside and around each scattering as the atomic-like orbital. On the other hand, because $\omega^{2}$ corresponds to the case where the electronic energy is higher than the maximum of the potential, there is an additional the host material. It means that resonant states for $\mathrm{AC}$ are states embedded in the continuum. This is an aspect of the problem not encountered in the electronic case.

\section{Conclusions}

In this work, we have investigated theoretically the

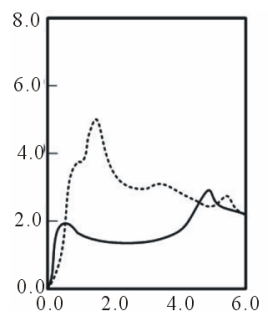

(a)

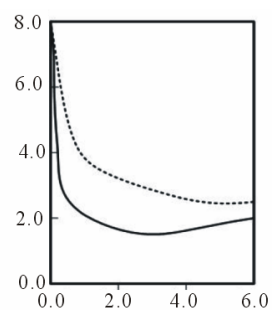

(b)

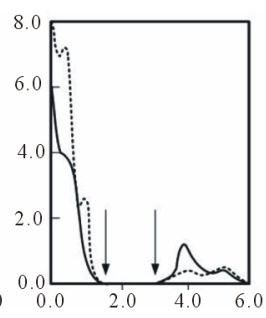

(c)
Figure 2. Dimensionless scattering cross sections for steel (a), rigid (b) cylinder embedded in air host. Panel (c): results by subtracting the amplitudes of (a) and (b). Solid curves: dimensionless scattering cross sections for longitudinal wave. Dashed curves: dimensionless scattering cross sections for transverse wave. propagation of acoustic waves in a binary $2 \mathrm{D}$ phononic crystal constituted of a square array of parallel, circular, steel cylinder in air resin matrix. We have limited the wave propagation to the plane perpendicular to the cylinders. The numerical calculations prove unambiguously the existence of absolute stop band independent of the direction of propagation of the acoustic waves. Besides the band gaps, one can establish some qualitative and even semiquantitative correspondences between the experimental and theoretical transmission spectra inside the pass bands. However, a more quantitative comparison would need to repeat such experiments with other samples (for instance to check the possibility of defects during the sample preparation, different thicknesses of the samples, etc.), in this respect, an analysis of the eigenvectors associated with the different modes would be also helpful for an understanding of the details of the experimental transmission spectra.

We extended the far field approach and presented transmissive and scattering properties of acoustic waves in finite-sized phononic band gap (PBG) material. This method make the calculating can be extremely simplified. We found that full band gap is created between well separated resonance states in which one can't achieve coherent jumping from a resonance state to a neighboring resonance states in analogy with the linear combination of atomic orbits in the electronic band structure; On the other hand, such that the propagation along the host material is inhibited. This results in full band gap appearing.

\section{References}

[1] M. S. Kushwaha, "Stop-Bands for Periodic Metallic Rods: Sculptures That Can Filter the Noise," Applied Physics Letters, Vol. 70, No. 24, 1997, pp. 3218-3220. doi:10.1063/1.119130

[2] H. Sanchis Alepuz, Y. A. Kosevich and J. Sanchez Dehesa, "Acoustic Analogue of Electronic Bloch Oscillations and Resonant Zener Tunneling in Ultrasonic Superlattices," Physical Review Letters, Vol. 98, No. 13, 2007, pp. (134301-1)-(134301-4).

doi:10.1103/PhysRevLett.98.134301

[3] M. Torres, F. R. Montero de Espinosa, D. García-Pablos and N. García, "Sonic Band Gaps in Finite Elastic Media: Surface States and Localization Phenomena in Linear and Point Defects," Physical Review Letters, Vol. 82, No. 15, 1999, pp. 3054-3057. doi:10.1103/PhysRevLett.82.3054

[4] D. Sutter Widmer, S. Deloudi and W. Steurer, "Prediction of Bragg-Scattering-Induced Band Gaps in Phononic Quasicrystals," Physical Review Letters, Vol. 75, No. 9, 2007, pp. (094304-1)-(094304-11). doi:10.1103/PhysRevB.75.094304

[5] M. Torres, F. R. Montero de Espinosa and J. L. Aragón, "Ultrasonic Wedges for Elastic Wave Bending and Split- 
ting without Requiring a Full Band Gap," Physical Review Letters, Vol. 86, No. 19, 2001, pp. 4282-4285. doi:10.1103/PhysRevLett.86.4282

[6] D. Garcia-Pablos, F. R. Montero de Espinosa, M. Torres, M. Kafesaki and N. García, "Theory and Experiments on Elastic Band Gaps," Physical Review Letters, Vol. 84, No. 19, 2000, pp. 4349-4352. doi:10.1103/PhysRevLett.84.4349

[7] J. O. Vasseur, P. A. Deymier, B. Djafari-Rouhani, L. Dobrzynski and D. Prevost, "Experimental and Theoretical Evidence for the Existence of Absolute Acoustic Band Gaps in Two-Dimensional Solid Phononic Crystals," Physical Review Letters, Vol. 86, No. 14, 2001, pp. 3012-3015. doi:10.1103/PhysRevLett.86.3012

[8] S. X. Yang, J. H. Page, Z. Y. Liu, M. L. Cowan, C. T. Chan and P. Sheng, Physical Review Letters, Vol. 88, No. 10, 2001, pp. (104301-1)-(104301-4).

[9] S. X. Yang, J. H. Page, Z. Y. Liu, M. L. Cowan, C. T. Chan and P. Sheng, Physical Review Letters, Vol. 93, No. 2, 2004, pp. (024301-1)-(024301-4).

[10] Z. Y. Liu, X. X. Zhang, Y. W. Mao, Y. Y. Zhu, Z. Y. Yang, C. T. Chan and P. Sheng, "Locally Resonant Sonic Materials," Science, Vol. 289, No. 8, 2000, pp. 17341736. doi:10.1126/science.289.5485.1734

[11] F. Cervera, L. Sanchis, J. V. Sánchez-Pérez, R. MartínezSala, C. Rubio, F. Meseguer, C. López, D. Caballero and J. Sánchez-Dehesa, Physical Review Letters, Vol. 88, No. 2, 2002, pp. (023902-1)-(023902-4).

[12] M. S. Kuswaha, Applied Physics Letters, Vol. 2, 1999, pp. 743-755.

[13] C. Goffaux and J. Sanchez-Dehesa, "Two-Dimensional Phononic Crystals Studied Using a Variational Method: Application to Lattices of Locally Resonant Materials," Physical Review B, Vol. 67, No. 14, 2003, pp. (1443011)-(144301-10). doi:10.1103/PhysRevB.67.144301
[14] Y. Y. Chen and Z. Ye, "Theoretical Analysis of Acoustic Stop Bands in Two-Dimensional Periodic Scattering Arrays," Physical Review E, Vol. 64, No. 3, 2001, pp. (036616-1)-(036616-6). doi:10.1103/PhysRevE.64.036616

[15] Y. Tanaka, Y. Tomoyasu and S. I. Tamura, Physical Review B, Vol. 62, 2000, pp. 7383-7395.

[16] R. M. White, Journal of the Acoustical Society of America, Vol. 30, No. 8, 1958, pp. 771-785.

[17] J. D. Jackson, “Classica Electrodynamics," Wiley, New York, 1975.

[18] M. M. Sigalas and E. N. Economou, Journal of Sound and Vibration, Vol. 158, No. 2, 1992, pp. 377-382.

[19] E. N. Economou and M. M. Sigalas, "Classical Wave Propagation in Periodic Structures: Cermet versus Network Topology," Physical Review B, Vol. 48, No. 18, 1993, pp. 13434-13438. doi:10.1103/PhysRevB.48.13434

[20] M. M. Sigalas and E. N. Economou, "Attenuation of Multiple-Scattered Sound," Europhysics Letters, Vol. 36, No. 4, 1996, pp. 241-246. doi:10.1209/epl/i1996-00216-4

[21] C. Kittel, "Introduction to Solid State Physics," Wiley, New York, 1971.

[22] R. D. Meade, K. D. Brommer, A. M. Rappe and J. D. Joannopoulos, "Electromagnetic Bloch Waves at the Surface of a Photonic Crystal," Physical Review B, Vol. 44, No. 19, 1991, pp. 10961-10964. doi:10.1103/PhysRevB.44.10961

[23] M. Kafesaki and E. N. Economou, "Interpretation of the Band-Structure Results for Elastic and Acoustic Waves by Analogy with the LCAO Approach," Physical Review $B$, Vol. 52, No. 18, 1995, pp. 13317-13331. doi:10.1103/PhysRevB.52.13317 